\title{
Operation of solid rockets in comparison with hybrid rockets during the STERN project
}

\author{
K. Schüttauf ${ }^{1}$ and A. Stamminger ${ }^{2}$ \\ Mobile Rocket Base (MORABA), German Aerospace Center (DLR), Oberpfaffenhofen, 82234 Wessling, Germany \\ K. Lappöhn ${ }^{3}$ \\ Space Administration, German Aerospace Center (DLR), 53227 Bonn, Germany \\ and \\ H. K. Ciezki ${ }^{4}$ and W. Kitsche ${ }^{5}$ \\ Institute of Space Propulsion, German Aerospace Center (DLR), Lampoldshausen, 74239 Hardthausen, Germany
}

In April 2012, the German Aerospace Center DLR launched a support program for students to develop, build and launch their own rockets over a period of three years. The program goes by the acronym STERN (STudentische Experimental-RaketeN).

The primary goal of the STERN program is to inspire students in the subject of space transportation through hands-on activities within a project structure, to motivate universities to supervise and support the student projects with the help of financial support and to increase the lecture activities in the field of launcher and propulsion systems. The program is supported by funds from the German Federal Ministry of Economics and Technology (BMWi) and managed by the DLR Space Administration. There are no limits regarding flight altitude or the propulsion systems used by the student teams. The students are free to develop, test and launch their own rocket, or purchase a commercial propulsion system. The main demands for each student project are that (1) the rocket shall reach a velocity of at least Mach 1 . Moreover (2)the rocket must have a telemetry system to transmit key trajectory and housekeeping data back to earth during flight and provide information to the students including the rocket altitude. (3) It must have a recovery system. Currently, eight German universities are participating in the STERN-program. In October 2015, the first launch campaign was scheduled in ESRANGE, Sweden. Two solid and two hybrid rockets were launched.

Ground and mission operation processes are highly complex and involve a wide variety of technologies. The operation of hybrid rocket motors requires a different kind of care and supervision in comparison to the procedures for a solid rocket motor. This paper describes launch procedures and shows that the vehicle design has a major impact on ground facilities, ground safety and operations.

\section{Introduction}

$\mathrm{T}$ HIS document provides an overview about the launch campaign at ESRANGE near Kiruna Sweden in October 2015 within the STERN program. In the following is a short introduction to the STERN program, the used rockets with their hybrid and solid rocket motors as well as the operational activities during the campaign special attention is paid to the operational differences in handling solid and hybrid propellants rockets. Detailed information about the rockets and their development are given in separate publications by the student teams ${ }^{1}$.

\footnotetext{
${ }^{1}$ MORABA STERN project manager, German Aerospace Center (DLR), Münchener Str. 20

${ }^{2}$ Team Leader of MORABA Business Development, German Aerospace Center (DLR), Münchener Str. 20

${ }^{3}$ STERN Programme Manager, German Aerospace Center (DLR), Königswinterer Str. 522-524

${ }^{4}$ Head Propellants Department, German Aerospace Center (DLR)

${ }^{5}$ Lampoldshausen Senior Test Leader, German Aerospace Center (DLR) 


\section{STERN program}

In April 2012, the German Aerospace Center DLR launched the STERN program, which is supported by funds from the Germen Federal Ministry of Economics and Technology (BMWi) and conducted by the DLR Space Administration.

The aim of the STERN program is to inspire students in the subject of space transportation through hands-on activities, to motivate universities to supervise and support the student projects with the help of financial, infrastructure and laboratory support and to increase the lecture activities in the field of launcher and propulsion systems.

The program is open for all German universities which offer aerospace engineering lectures and offer students the possibility to develop, build, test and fly their own rockets in university teams. The focus is on the development of the complete vehicle system within three years.

The student's shall develop their knowledge in the following disciplines:

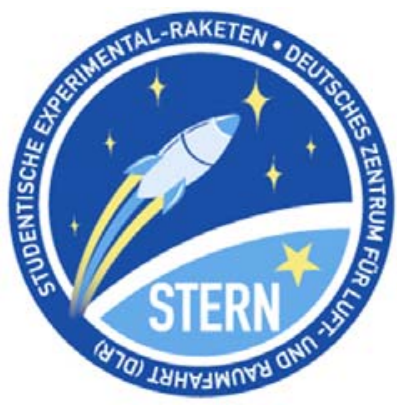

Figure 1. Logo of STERN program

- Rocket propulsion and space thrust systems

- Aerodynamics, mechanics and lightweight structures

- Performance calculation, trajectory optimization, mass and center of gravity calculations

- Application of professional tools (for example CAD, CFD, FEM)

- Wind tunnel investigations

- Manufacturing and integration of parts

- Testing of the rocket and its subsystems, and

- Launch of a sounding rocket

- Flight dynamics

From the beginning of the project until the launch of the rocket, the students have to conduct several reviews in which they will have to present the current status of their work to a review team consisting of DLR Space Administration, DLR experts from Mobile rocket base (MORABA) and the DLR Institute of Space Propulsion, but also further external experts if required. This aims to increase the chance of achieving the mission goal of the student teams and improve safety during development and launch of the vehicle

The requirements for participation in the STERN program are

1. Formal requirements

- German universities focusing on aerospace, particularly on launcher aspects. Teaching content at the university must be linked to the project.

- Conduct of reviews including the participation of minimum one reviewer from DLR MORABA, DLR Institute of Space Propulsion and the DLR Space Administration, respectively

2. Technical requirements

- Minimum apogee of $3 \mathrm{~km}$ and minimum velocity of the speed of sound

- Recovery system for the rocket, and

- Telemetry unit to transmit the most important trajectory data (acceleration, velocity, altitude and GPSposition) during flight to ground

\section{Description and comparison of hybrid and solid rocket motors}

A solid rocket motor consists of a motor casing in which the solid propellant is embedded and bonded to the wall. The solid propellant contains both fuel and oxidizer components, which are mixed homogeneously. Due to this fact solid propellants are typically rated as explosives. During operation, the solid propellant burns at its free surface, which regresses during the motor operation time. The produced hot gases flow through the thrust nozzle and are accelerated. Specific impulses of typical propellants are approximately in the range of $200-260$ s for combustion pressures of 70 bar and pressure (expansion) ratios of 70:1.

A conventional hybrid rocket motor consists of a combustion chamber in which a solid fuel is embedded and a tank with a liquid oxidizer. Due to the separation of fuel and oxidizer this motor type is not typically rated as an 
explosive. Specific impulses for conventional propellant combinations are in the range of approximately 230 - $270 \mathrm{~s}$ for the same thruster conditions as mentioned above.

Comparing solid rocket motors with hybrid rocket motors it can be said that solid rocket motors have the highest thrust density of all up to now known chemical propulsion motor types. The solid rocket motor has simple handling and storage characteristics because it has manufactory separate and then stored until they are brought to the launch platform. Thus it can be used instantaneously if they are needed. A hybrid rocket, however, will be fueled on the launch pad. As solid rocket propellants are typically rated as explosives or are in the hobby range as pyrotechnic devices, this demand special care, instruction and handling. They are simpler in the design because they have no feeding system for the oxidizer as a hybrid rocket motor. The solid rocket motor operation time is also typically shorter. Hybrid rocket motors are capable of a thrust variation free on demand, while the thrust profile of solid rocket motors is fixed by the propellant grain geometry. The simpler handling and safety characteristics of hybrids make them attractive for education of student groups. More detailed information about the characteristics of both propulsion types is given in References [2, 6, and 7].

\section{Overview of the student rockets}

Three of the university teams attended the lauch campaign in October 2015, with four rockets in total. The launches took place at the SSC ESRANGE Space Center in Kiruna, Sweden. All rockets, i.e. two hybrid rockets and two identical solid rockets, could be launched within the scheduled two weeks campaign. A short overview of the student rockets is given in the following. The authors assume that the student groups will present their rockets and the flight campaign in own publications in more detail than here.

\section{A. DECAN SHARK I and II}

"DECAN - Deutsche CanSatHöhenrakete" is the designation of the two-stage sounding rocket project of the Technical University of Berlin. However it was decided to launch only the Engineering and Qualification Model (EQM) and Flight Model (FM) of the upper stage at ESRANGE. Both rockets were identical except one contained a telemetry payload and the other one a dummy payload. The predicted apogee of the DECAN SHARK rockets was 6.5 $\mathrm{km}$. The solid rocket motor is a commercially available class $\mathrm{N}$ motor

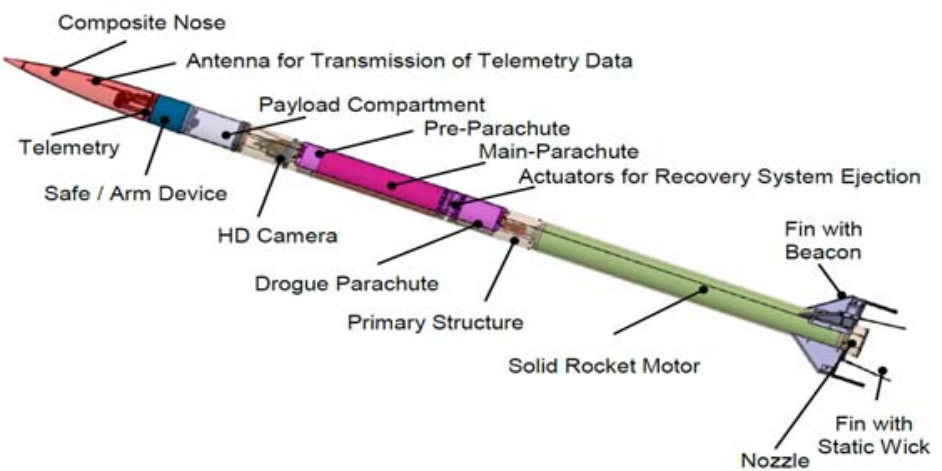

Figure 2. System of the SHARK rocket for high power rocketry with level 3 certification. Its propellant is an aluminum / ammonium perchlorate composite. The project was performed under professional supervision based on ECSS standards and was supported by the Aerospace Institute of the Technical University of Berlin. Detailed information about the development of the rocket and the motor is given in reference [3].

DECAN SHARK I was launched from the Medium Range Launcher (MRL) at 13:55UTC on October 27 and DECAN SHARK II was launched from the MRL as well at 08:55UTC on October 29. Before take-off of SHARK I, an unexpected long ignition delay of approximately 8 seconds of the rocket motor was observed. The reason

\begin{tabular}{|l|l|}
\hline Item & \\
\hline Length & $2881 \mathrm{~mm}$ \\
\hline Weight & $25 \mathrm{~kg}$ \\
\hline Thrust (nominal) & $2.0 \mathrm{kN}$ \\
\hline Max acceleration & $14 \mathrm{~g}$ \\
\hline Apogee (predicted) & $6.5 \mathrm{~km}$ \\
\hline Burning time & $6.9 \mathrm{sec}$ \\
\hline
\end{tabular}
for this was the low temperature of the rocket motor by the low ambient temperature during the extended countdown. The gas generator inside the combustion chamber needed more time to generate the required hot gas in order to provide the ignition temperature for the propellant grain. After the ignition of the solid motor, the rocket left the launcher and a stable flight trajectory was observed. It can be assumed that the integrity of the rocket structure was ensured during the entire flight. Furthermore, telemetry data was received during the flight and a maximum 
altitude of 5,556 m was reported. Moreover, a maximum speed of $401 \mathrm{~m} / \mathrm{s}$ (Mach 1.2) was measured by the sensors of the telemetry system. The triggering of the drogue parachute was reported by the telemetry system, as well. However the recovery system malfunctioned. It was observed that the rocket descended without any parachute and must have crashed into the ground.

The DECAN SHARK II rocket had a stable flight trajectory as well and reached an altitude of 5,703 m. A maximum speed of $414 \mathrm{~m} / \mathrm{s}$ (Mach 1.2) and an acceleration of $103 \mathrm{~m} / \mathrm{s}^{2}(10 \mathrm{~g})$ were measured. The triggering of the drogue and main parachute was reported from the telemetry system. It was observed that the drogue parachute was ejected; however the main parachute malfunctioned. Thus, the vehicle crashed into the ground as well. ${ }^{1,3}$

\section{B. HyEnD HEROS}

The STERN program enables the HyEnD student group (Hybrid Engine Development) at the University of Stuttgart to expand its field of work and to broaden their experience of engine development by building a sounding rocket. The team was supervised and supported by the Institute of Space Systems. Since the start of the project, several hybrid rocket engines were designed, built and ground tested. The goal of HyEnD's sounding rocket was to reach a minimum altitude of around 20 $\mathrm{km}$, with a top speed greater than Mach 2.5 , whereas a hybrid rocket engine of 10000 Newton thrust shall be realized. The name of the rocket is HEROS. Detailed information about the development of the rocket and the motor is given in reference ${ }^{8}$.

In the hybrid rocket motor $\mathrm{N}_{2} \mathrm{O}$ is used as the liquid oxidizer and paraffin as the solid fuel. The $\mathrm{N}_{2} \mathrm{O}$ was used without
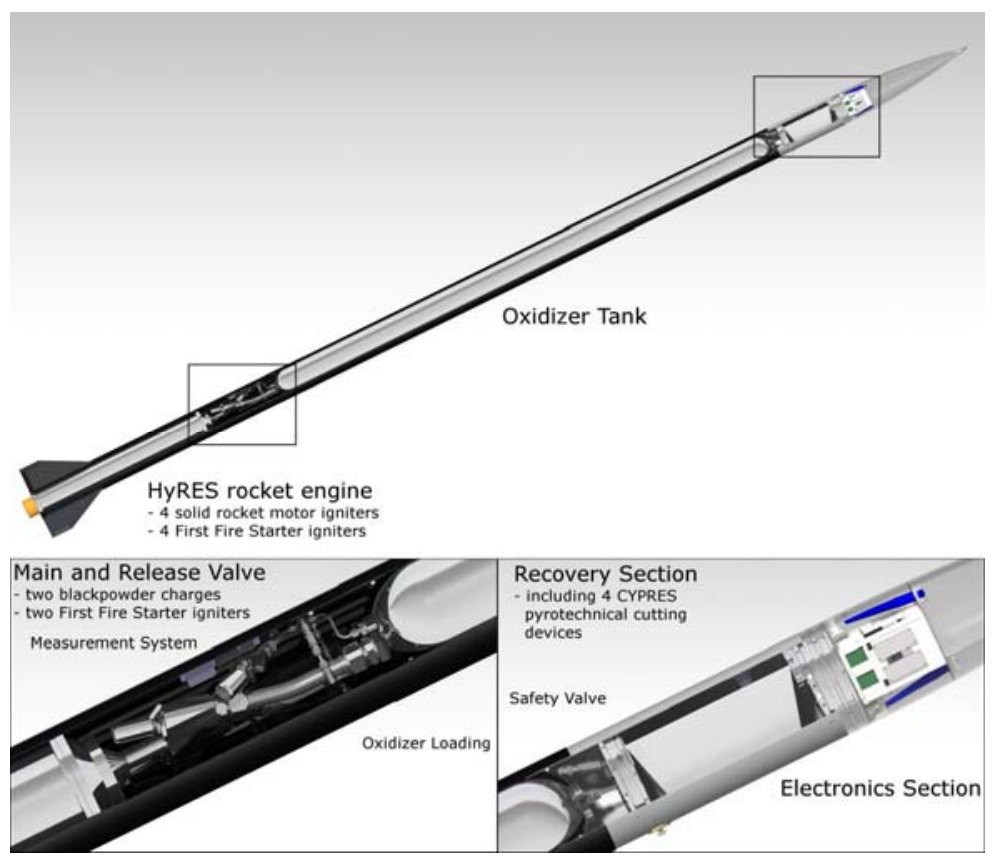

Figure 3. System of the HEROS external pressurization in the rocket in blow down mode. During the flight, the telemetry unit should send trajectory data to the ground station. Additionally a flight computer was implemented to store telemetry data, tank pressure and burning chamber pressure to a memory card. The flight computer also triggered the recovery system, which consisted of a drogue chute that should be ejected at apogee. The main chute should be triggered at a certain lower altitude.

HEROS was launched from the MRL at 14:45UTC on October 22. During the hot countdown some problems occurred, but this could not be identified at this time. The post flight analysis gave the hint that the air

\begin{tabular}{|l|l|}
\hline Item & \\
\hline Length & $7500 \mathrm{~mm}$ \\
\hline Weight & $177 \mathrm{~kg}$ \\
\hline Thrust (nominal) & $10 \mathrm{kN}$ \\
\hline Max acceleration & $7 \mathrm{~g}$ \\
\hline Apogee (predicted) & $>20 \mathrm{~km}$ \\
\hline Burning time & $25 \mathrm{sec}$ \\
\hline
\end{tabular}

Table 2: Characteristics of HEROS heating on the ground for the rocket seemed not to be sufficient which leaded probably to too low temperatures inside the rocket. This could be deduced after launch from the on-board acceleration data of the rocket, which indicates a thrust much lower than the design value. The design range of the temperature for the nitrous oxide during operation was $25^{\circ} \mathrm{C} \pm 5^{\circ} \mathrm{C}$. It is likely that the temperature was below $15^{\circ} \mathrm{C}$. This resulted in a much lower $\mathrm{N}_{2} \mathrm{O}$ pressure than what was tested on the ground test bench. A lack of on-board sensor data was the reason that this was not identified during the countdown. During the flight, the low nitrous oxide temperature caused very low tank and thus feeding pressures. Therefore, the engine pressure was also much lower than nominal. These low pressure conditions could have caused strong low frequency instabilities in the engine's combustion process, which can be seen both in the video material of the launch and in the acceleration data of the telemetry unit. Detailed failure 
investigations are still ongoing and a Failure Analysis Review is planned. The current state of knowledge is that the engine structure failed due to these instabilities and hence caused a rupture of the side wall. The outflowing hot gas increased the diameter of the hole and also caused the rocket to rotate faster. The lower $50 \mathrm{~cm}$ of the rocket including the fins broke off. At this point the rocket started tumbling heavily and the high aerodynamic drag slowed it down immediately, resulting in a low apogee of only $2 \mathrm{~km}$. The top speed was around $290 \mathrm{~m} / \mathrm{s}$, which is $\sim$ Ma 0.9 . When the rocket started tumbling with this high speed at a high rate, the centrifugal forces were too high for the recovery bay cover. It broke and released the parachutes. At this high speed, the parachute lines broke and therefore the rocket landed without parachutes. The vehicle without the fins was falling in a horizontal position, reducing the impact speed to $\sim 40 \mathrm{~m} / \mathrm{s}$. Therefore all the flight computers were still intact and the flight data was available. Currently, an improved version of the HEROS rocket is planned to be built and launched in October 2016. Major improvements will include an increased number of pressure and temperature sensors on the rocket. ${ }^{1,2}$

\section{ERIG FAUST}

The ERIG is an association at the Technische Universität Braunschweig (Institute of Aerospace Systems ILR). As a STERN participant, the ERIG is developing a research hybrid rocket and a new hybrid rocket engine within the Leonis project. The rocket Faust was powered by a hybrid rocket motor called HYDRA3X. The HYDRA3X engine consists of a solid HTPB (Hydroxylterminated polybutadiene) / aluminum powder mixture and liquid oxidizer (nitrous oxide) and was planned to deliver $1.25 \mathrm{kN}$ of thrust, which should allow the rocket to reach altitudes in the order of $5 \mathrm{~km}$. The rocket FAUST was used with external helium pressurization. Furthermore, ERIG was designing a

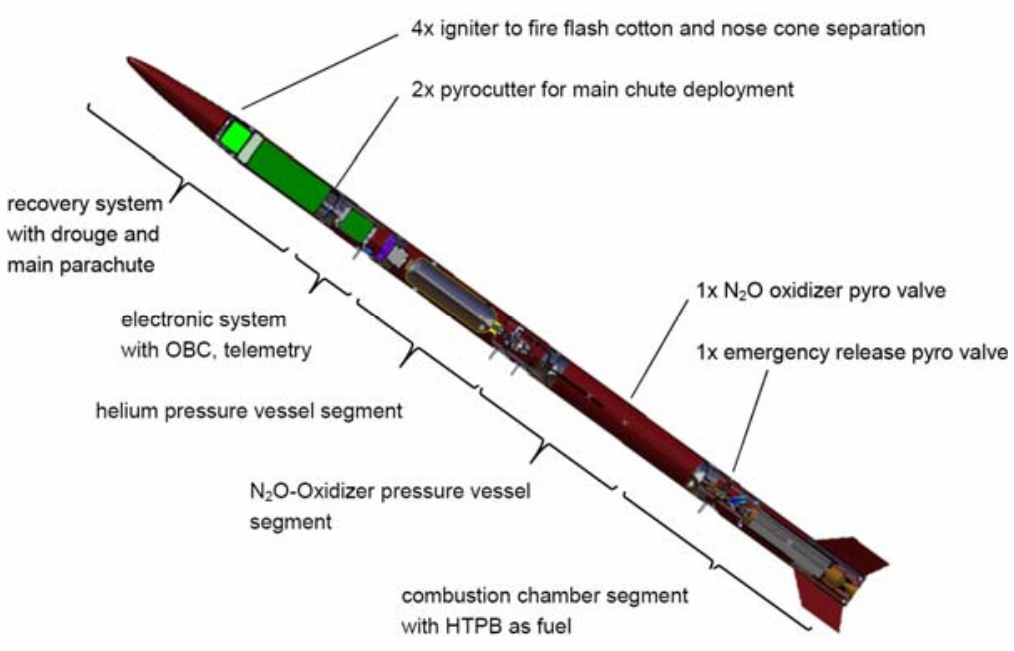

Figure 4. System of the FAUST

telemetry platform with the help of an inertial measurement unit (IMU) and navigation data via GPS, as well as a new flight simulation tool called ExRaS (ExperimentalRaumfahrt-Simulation). This allows for individual simulation runs with different configurations and the estimation of key parameters such as the maximum altitude and velocity. By including online weather data, it is possible to perform a complete flight simulation and thus predict where the vehicle will touch down. Detailed information about the development of the rocket and the motor is given in Reference [4].

FAUST was launched from the team own launcher at 08:45UTC on

\begin{tabular}{|l|l|}
\hline Item & \\
\hline Length & $3201 \mathrm{~mm}$ \\
\hline Weight & $24 \mathrm{~kg}$ \\
\hline Thrust (nominal) & $1.25 \mathrm{kN}$ \\
\hline Max acceleration & $3.65 \mathrm{~g}$ \\
\hline Apogee (predicted) & $6.32 \mathrm{~km}$ \\
\hline Burning time & $10 \mathrm{sec}$ \\
\hline
\end{tabular}

Table 3: Characteristics of FAUST

October 22. The engine worked according to design and latest tests. At this time no malfunction could be detected. However, the rocket crashed into the ground due to a malfunction in the recovery system. Based on the flight data it is assumed that the drogue chute deployed as planned but the velocity was far higher than expected. Even the integrated damper was not able to alleviate the overload. Because of this, the drogue chute ripped off. This theory is supported by the recovered and unrecovered parts. The main chute was found inside the rocket whilest the drogue chute was missing. The high apogee velocity might have been by the low launch angle of 80 degrees. This was not taken into account when the recovery system was designed. Nevertheless, the electronics performed as planned. No anomalies were detected during launch sequence and flight. However, due to the harsh impact, no flight data could be recovered from the on board memory. Only data transmitted during flight was available. Furthermore, the structural components of the rocket performed as planned during the flight. ${ }^{1,4}$ 


\section{Operations}

\section{A. Launch preparation}

For the launch preparation and operations phase the responsibility for each mission resided with the STERN teams. MORABA coordinated and managed the mission activities at the launch site. During launch preparation, the launcher, rocket, ground support equipment, telemetry station of each team were transported to the launch range, assembled, adjusted and tested. A so called procedure meeting was conducted regularly between range and STERN teams. The aim is to raise ensure the safe conduct of propellants and igniters, which are governed by explosive law, and other dangerous items and fluids. During the procedure meeting, all participants reviewed all relevant Safe/Arm conditions, reading the countdown procedure step by step and demonstrate the plan of fluids.

A practice test countdown was performed in order to check the integrity of the sequence and to exercise persons involved in launch activity and operations. A test countdown is an important measure to harmonize operations between the different teams involved in the launch. The operational teams during a STERN Countdown were Operations (SSC), Vehicle (SSC), Rocket system (STERN team), Safety (SSC), Flight control (SSC), as well as Communication (SSC). Depending on the type of vehicle used, STERN countdowns can start from 2 to 8 hours before launch time. During this test, the

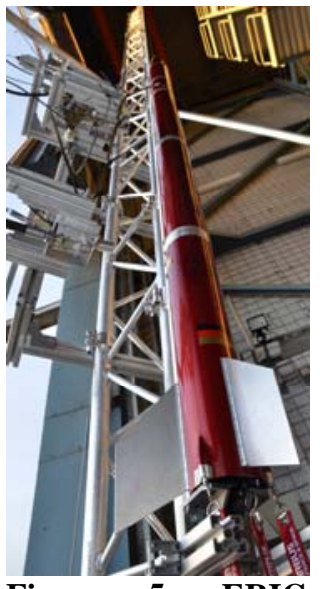

Figure 5: ERIG fueling arm ignition chain was not fully closed and the fueling of the tanks was not processed (see ERIG rocket on launch rail Figure 5). The successful completion of the test countdown served as input for the Flight Readiness Review (FRR). The Flight Readiness Review was held on the launch range after completion of all preparatory work and prior to the hot countdown. All parties involved report the status of their contribution. Finally, all systems had to be declared ready for flight by all representatives of the teams involved.

The nominal lift-off time in Sweden has to be planned between 05.00 and 16.00 LT. This time span is determined by the rocket preparation time, hold requirements and the time span of daylight. The maximum launch window duration is 11 hours. The decision to start the countdown is taken at a weather briefing immediately before the planned start of countdown. If the weather conditions are unsuitable for launching the vehicle, the launch will be delayed until the flight conditions are fulfilled. A new detailed countdown procedure is provided by the range for each countdown. The process flow diagram of the countdown is shown in Figure 6.

A recovery of the STERN rockets was foreseen. Every (major) part of the rocket should be returned back to the range. During the flight, the rocket trajectory should be tracked by the STERN teams. During the descent of the rocket, the prediction on the impact point coordinates were reported.

After each flight all parties involved present a short performance report of their systems or field of operation. These inputs are consolidated in a general short campaign report. Any possible deviations from nominal were reported and necessary action items were issued. 


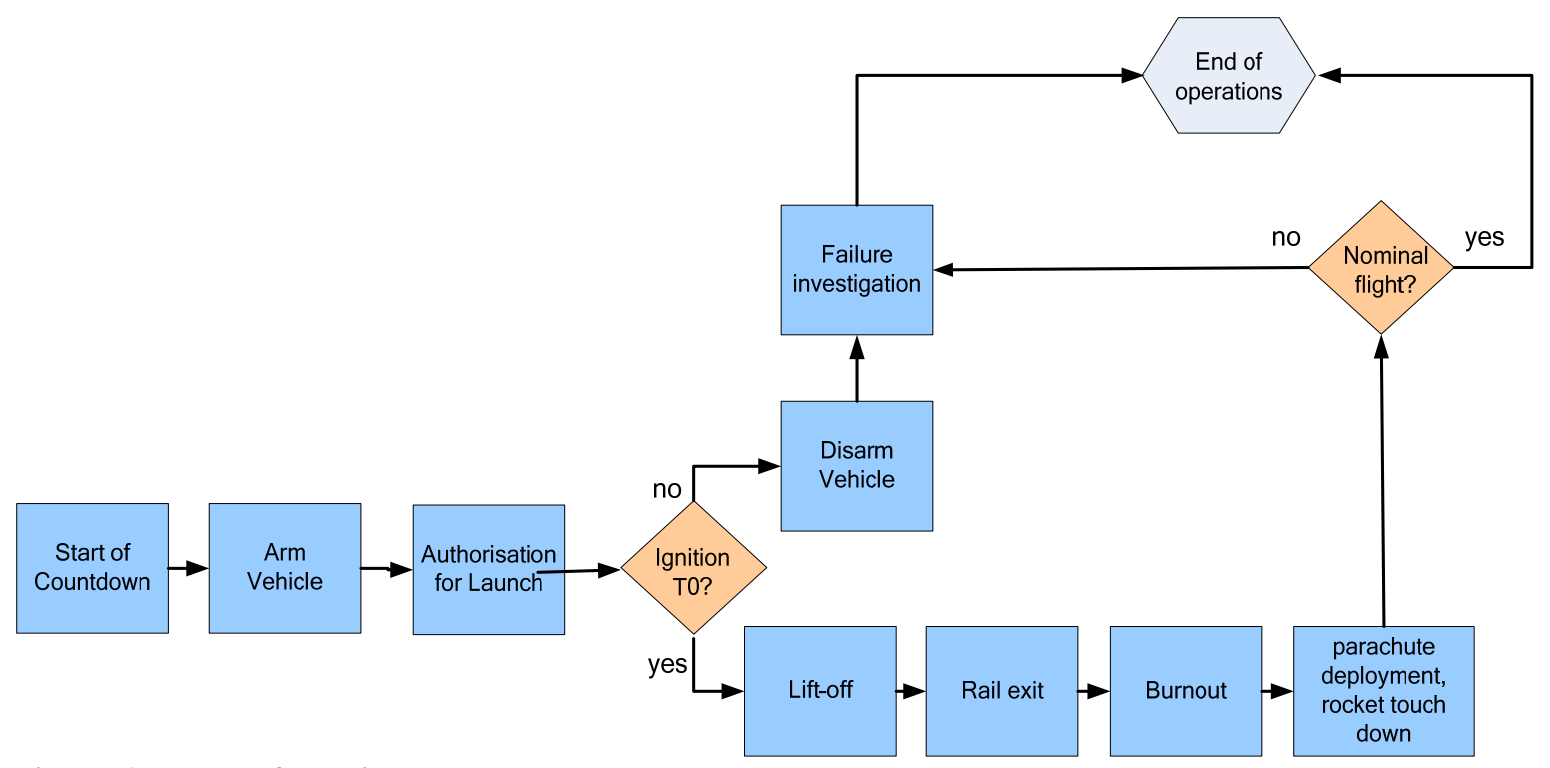

Figure 6: Process flow diagram countdown

\section{Solid rocket (DECAN)}

The motors arrive at Esrange launch site in a pre-assembled configuration. The DECAN team assembled the rocket motor and related systems according to the dedicated work (range) instructions and under supervision of the launch site personnel and members of the DLR review team. Preparations for the motor include inspections and system checks. The payload assembly was performed externally. Afterwards, a subsystem test, assembly of launch rail, rigging of the payload and motor umbilical were conducted. An extraction test of the rocket from the rail at the end of the installation demonstrates to payload and range representatives the proper function and that the fin travelling area is free of any objects. Figure 7 shows the launch preparation steps and Figure 8 a process flow diagram of solid rocket countdown

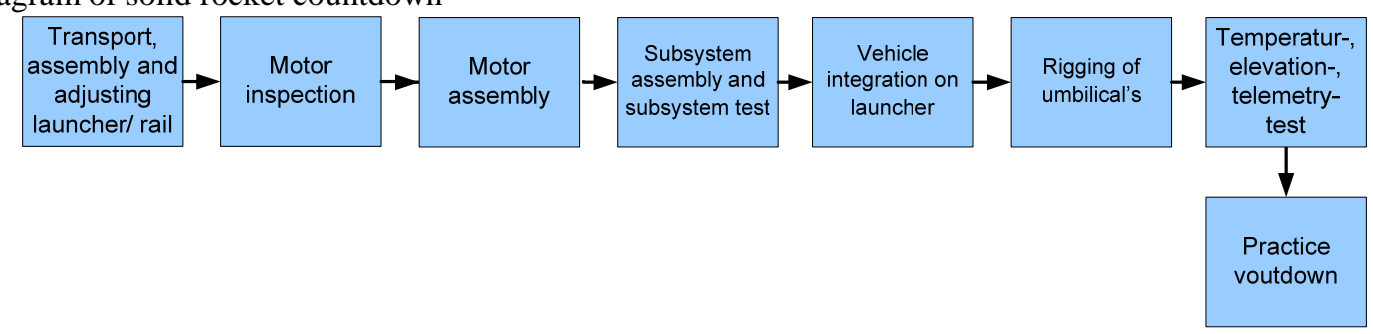

Figure 7: Solid launch vehicle preparation

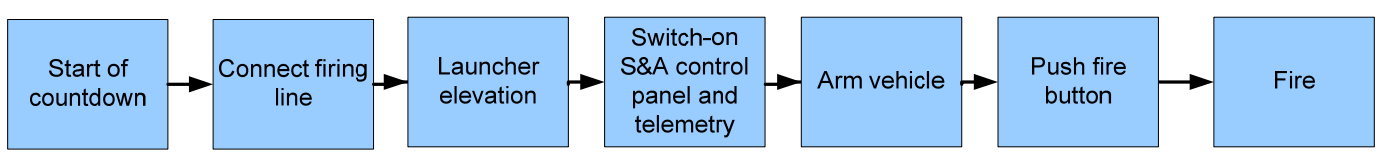

Figure 8: Process flow diagram solid rocket countdown

\section{Hybrid rocket (HyEnD and ERIG)}

The rocket sections are transported partially preassembled to increase the processing speed at the launch site. Because the rocket compartments were temperature critical, the Ground Support Equipment (ERIG) and the rocket (HyEnD) had to be covered ("boxed") with polystyrene. The gas bottles have been delivered to the range. The paraffin fuel is not classified as a solid propellant and is not dangerous to ground personnel. It was delivered by normal transportation. Afterwards the propulsion system was connected and assembled. It had to be checked that all pipes and valves are clean to avoid complications with $\mathrm{N}_{2} \mathrm{O}$. Next were conducted mounting of the launch equipment (like launch rail at the MRL (HyEnD), building up their own launching device (ERIG)), performing of pressure, tightness and leakage test. The process chart of the hybrid launch vehicle preparation is shown in Figure 9. 


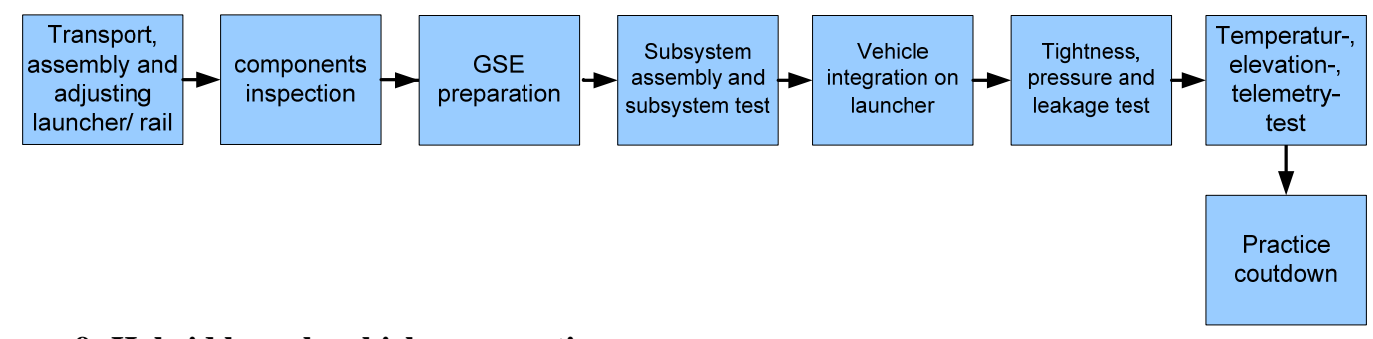

\section{Figure 9: Hybrid launch vehicle preparation}

In Figure 10 the countdown process flow diagram of a hybrid rocket during the STERN program is shown. For ERIG: To arm the vehicle the power supply of the ground support electronics was activated. After a few seconds the controller was booted, so that it was in a defined state. The safety switch box at the launch site was set to 'armed'. The EEDs were connected to the electronics but they were still shorted and grounded within the electrical enclosure. Then the staff left the launch site. In the next step the key switch was activated and the rocket was fueled with nitrous oxide and helium. The fueling process was controlled remotely from the launch control site. After the fueling process the fueling pipes were disconnected from the rocket. The arming switch was activated. The system was armed for 20 seconds. The rocket was then launched by pressing the fire-button. For HyEnD the countdown process was quite similar, except the pyro valves were armed by connecting their cables to the provided firing lines and then removing the S/A devices.

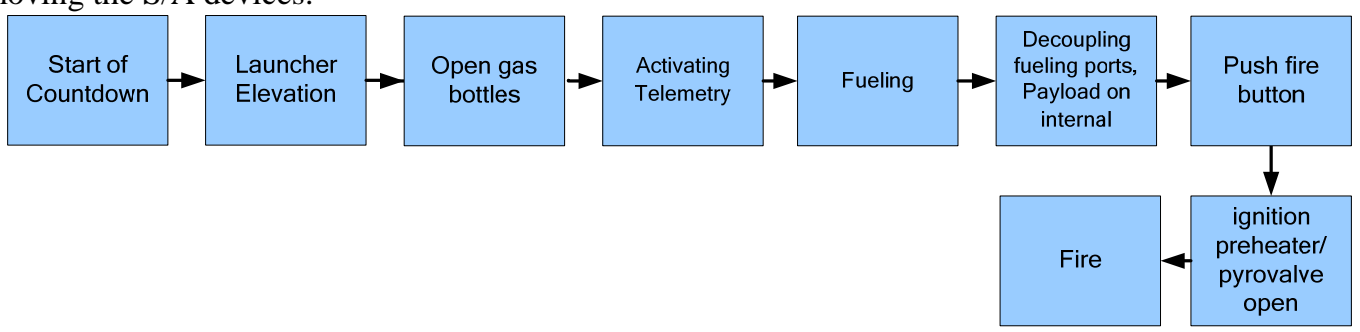

Figure 10: Process flow diagram hybrid rocket countdown

3. Comparison

\begin{tabular}{|l|l|l|}
\hline & Solid rocket (DECAN) & $\begin{array}{l}\text { Hybrid rocket (ERIG } \\
\text { / HyEnD) }\end{array}$ \\
\hline Planned countdown period [h] & 1 & 1.25 \\
\hline Average time between ignition and lift off [s] & 8 (too low temperatures) & 2.5 \\
\hline Pre preparation duration [days] & 4 & 5 \\
\hline Boxing & no & yes \\
\hline $\begin{array}{l}\text { Personal risk [ground safety] for arming at launcher during } \\
\text { countdown }\end{array}$ & medium & low \\
\hline$\%$ of matching test- and hot Countdown & $95 \%$ & $80 \%$ \\
\hline
\end{tabular}

Table 4: Operational difference between the conducted launches ${ }^{1-4}$

\section{B. Mission Post Processing}

The teams are responsible for providing post flight processing and analysis of raw technical data recovered from rocket missions. This data is provided to the DLR Space Administration. Using all collected mission data, a report about the flight and the performance of all subsystems was issued. Each mission post processing was concluded by a lessons learned meeting in order to evaluate the overall mission success, identify and assess non-conformances and the potential for improvements.

\section{Conclusion \& Outlook}

The experience and results from the first STERN launch campaign demonstrated a very good performance of the first student rockets and the good approach for student support by the STERN program strategy. All rockets were launched successfully on the first attempt (see Figure 11). Except one, all rockets reached the nominal speed and altitude and a large volume of flight data was collected from each flight. As weak point the recovery system was identified in all rocket systems. 

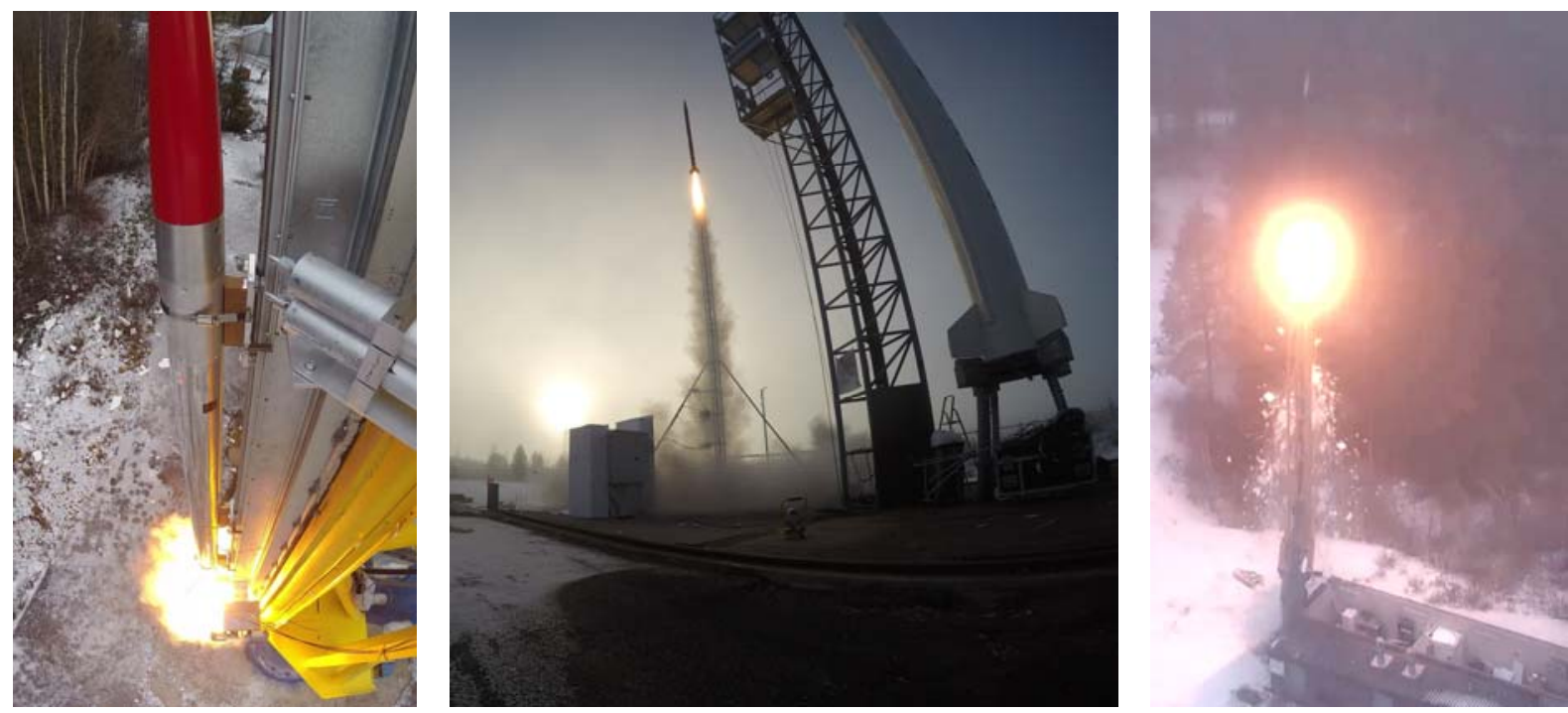

Figure 11: Launch of SHARK I, FAUST and HEROS (f.l.t.r.)

The preparation and launch procedures of the student groups were well set up and conducted in conformance to the professional requirements of the team on ESRANGE launch site. Especially the remote fueling of rockets shortly before launch was new to ESRANGE, but could be well integrated in the launch procedures. The students demonstrated their ability to fulfill the high standards of safety concerning the handling of pyrotechnical elements and solid rocket motors as well as the safety aspects for managing a remote controlled fueling of the rocket.

It could be seen that, due to their different system architecture, the handling and the operation of the STERN hybrid rockets and the STERN solid rockets showed some differences. For the hybrids rockets the launch preparation time was longer and the workload during the countdown was higher. The way how the test countdown and hot countdown were conducted is for a solid rocket more similar, because the fueling process of hybrids with the liquid oxidizer demands typically amongst others a defueling after the test countdown. The personal risk for the final arming process of the rocket at the launch pad during the hot countdown was higher for the solid rocket teams as for the hybrid rocket teams. The solid propellant is premixed and thus rated as explosive, while fuel and oxidizer in a hybrid rocket are separately stored, so that the risk of an explosion is significantly reduced.

The demanding aspect of this program is that it requires knowledge of a large variety of engineering topics and involves operational process as well. This whole range of different topics makes the STERN program very interesting and enhances various skills of the participating students and partners. The launch of another two hybrid rockets in the STERN program is scheduled for April 2016. After formally closing the projects, the universities will have the opportunity to apply for a new project within a follow-up program STERN II, which is currently in planning stage and which could be started in January 2017. It is of highest importance to secure a continuation of this programme, because the education of students is an ongoing process. 
BAM

DECAN

DLR

EED

Esrange

ExRaS

FRR

GPS

GSE

HTPB

HyEnD

IMU

LAM

MORABA / MRB

MRL

RB

SCC

STERN

\section{Abbreviations}

Federal Institute for Materials Research and Testing

Deutsche CanSat-Höhenrakete (engl. German CanSat sounding rocket)

Deutsches Zentrum für Luft- und Raumfahrt (engl. German Aerospace Center)

Electric explosive device

European Space Range

ExperimentalRaumfahrt-Simulation (engl. Experimental aerospace simulation)

Flight Readiness Review

Global Positioning System

Ground Support Equipment

Hydroxyl-terminated polybutadiene

Hybrid Engine Development

Inertial measurement unit

Lampoldshausen

Mobile Raketenbasis (DLR) (engl. Mobile rocket base)

Medium Range Launcher

Space Operations and Astronaut Training

Swedish Space Corporation

Studentische Experimental-Raketen

\section{References}

${ }^{1}$ Töyrä, M., “STERN Campaign Report”, REK221-E66, 2015

${ }^{2}$ Kobald, M., Schmierer, C., Fischer, U., Tomilin, K., Petrarolo, A., "The HyEnD STERN Hybrid Sounding Rocket Project" in: EUCASS Book Progress in Propulsion Physics; Bonnal, C.; Calabro, Galfetti, L. \& Maggi, F. (Eds.), 2015, submitted for publication

${ }^{3}$ Schmid M, Fleischer P., Sliwowski F.,DECAN (Deutsche CanSat-Höhenrakete) - A Sounding Rocket Project at TU Berlin, in: EUCASS Book Progress in Propulsion Physics; Bonnal, C.; Calabro, Galfetti, L. \& Maggi, F. (Eds.), 2015, submitted for publication

${ }^{4}$ May S., Poppe G., Pöppelmann M., Sültrop H. P., Vörsmann P., Development of a Supersonic Research Rocket with Hybrid Rocket Engine, in: EUCASS Book Progress in Propulsion Physics; Bonnal, C.; Calabro, Galfetti, L. \& Maggi, F. (Eds.), 2015, submitted for publication

${ }^{5} \mathrm{NN}$, NASA, “CHEMICAL ROCKET LAUNCHER", Space Settlements [online webpage], URL: http://settlement.arc.nasa.gov/Nowicki/SPBI101.HTM [cited 18 march 2016]

${ }^{6}$ Ciezki, K. K., Negri, M., Gernoth, A., "Advanced Liquid and Gelled Propellants for Rocket and Ramjet Propulsion,” Int. Journal of Energetic Materials and Chemical Propulsion, 2015, Vol. 14, No. 2, pp. 85-123.

${ }^{7}$ Ciezki, H. KNaumann, K. W., "Some Aspects on Safety and Environmental Impact of German Green Gel Propulsion Technology,” Propellants, Explosives, Pyrotechnics, 2016, accepted for publication 